\title{
Article \\ A Note on the Abelian Complexity of the Rudin-Shapiro Sequence
}

\section{Xiaotao Lü and Pengju Han *}

check for

updates

Citation: Lü, X.; Han, P. A Note on the Abelian Complexity of the Rudin-Shapiro Sequences. Mathematics 2022, 10, 221. https:// doi.org/10.3390/math10020221

Academic Editor: Dumitru Baleanu

Received: 17 December 2021

Accepted: 11 January 2022

Published: 12 January 2022

Publisher's Note: MDPI stays neutral with regard to jurisdictional claims in published maps and institutional affiliations.

Copyright: (C) 2022 by the authors. Licensee MDPI, Basel, Switzerland. This article is an open access article distributed under the terms and conditions of the Creative Commons Attribution (CC BY) license (https:// creativecommons.org/licenses/by/ $4.0 /)$.
College of Science, Huazhong Agricultural University, Wuhan 430070, China; xiaotaoLv@mail.hzau.edu.cn

* Correspondence: hanpengju@mail.hzau.edu.cn

Abstract: Let $\{r(n)\}_{n \geq 0}$ be the Rudin-Shapiro sequence, and let $\rho(n):=\max \left\{\sum_{j=i}^{i+n-1} r(j) \mid i \geq\right.$ $0\}+1$ be the abelian complexity function of the Rudin-Shapiro sequence. In this note, we show that the function $\rho(n)$ has many similarities with the classical summatory function $S_{\mathbf{r}}(n):=\sum_{i=0}^{n} r(i)$. In particular, we prove that for every positive integer $n, \sqrt{3} \leq \frac{\rho(n)}{\sqrt{n}} \leq 3$. Moreover, the point set $\left\{\frac{\rho(n)}{\sqrt{n}}: n \geq 1\right\}$ is dense in $[\sqrt{3}, 3]$.

Keywords: Rudin-Shapiro sequence; abelian complexity; growth order; dense property

\section{Introduction}

In this note, we are concerned with the abelian complexity $\rho(n)=\max \left\{\sum_{j=i}^{i+n-1} r(j) \mid\right.$ $i \geq 0\}+1$ of the Rudin-Shapiro sequence $\mathbf{r}$. The Rudin-Shapiro sequence $\mathbf{r}=r(0) r(1) \cdots \in$ $\{-1,1\}^{\mathbb{N}}$ is given by the following recurrence relations:

$$
r(0)=1, r(2 n)=r(n), r(2 n+1)=(-1)^{n} r(n)(n \geq 0) .
$$

The Rudin-Shapiro sequence $\mathbf{r}$ is a typical 2-automatic sequence [1]. It has been proved in [2] that the sequence $\rho(n)$ satisfies $\rho(1)=2, \rho(2)=3, \rho(3)=4$ and for every $n \geq 1$,

$\rho(4 n)=2 \rho(n)+1, \rho(4 n+1)=2 \rho(n), \rho(4 n+2)=\rho(n)+\rho(n+1), \rho(4 n+3)=2 \rho(n+1)$.

Let $\mathbf{w}=w(0) w(1) w(2) \cdots$ be an infinite sequence with $w(i) \in \mathbb{Z}$ for every $i \geq 0$. There are many papers focusing on the summatory function $S_{\mathbf{w}}(n):=\sum_{j=0}^{n} w(j)$. In [3-5], Brillhart and Morton studied the summatory function $S_{\mathbf{r}}(n):=\sum_{i=0}^{n} r(i)$ of the RudinShapiro sequence. The sequence $S_{\mathbf{r}}(n)$ satisfies $S_{\mathbf{r}}(0)=1, S_{\mathbf{r}}(1)=2, S_{\mathbf{r}}(2)=3, S_{\mathbf{r}}(3)=2$ and for every $n \geq 1$,

$$
S_{\mathbf{r}}(4 n)=2 S_{\mathbf{r}}(n)+r(n), S_{\mathbf{r}}(4 n+1)=S_{\mathbf{r}}(4 n+3)=2 S_{\mathbf{r}}(n), S_{\mathbf{r}}(4 n+2)=2 S_{\mathbf{r}}(n)+(-1)^{n} r(n) .
$$

In detail, Brillhart and Morton proved that for every $n \geq 1$,

$$
\sqrt{3 / 5} \leq \frac{S_{\mathbf{r}}(n)}{\sqrt{n}} \leq \sqrt{6}
$$

and $\left\{\frac{S_{\mathbf{r}}(n)}{\sqrt{n}}: n \geq 1\right\}$ is dense in $[\sqrt{3 / 5}, \sqrt{6}]$. In [6], Lafrance, Rampersad and Yee introduced a Rudin-Shapiro-like sequence $(l(n))_{n \geq 0}$ which satisfies $l(0)=1$ and for every $n \geq 0$,

$$
l(4 n)=l(n), l(4 n+1)=l(2 n), l(4 n+2)=-l(2 n) \text { and } l(4 n+3)=l(n) .
$$

They studied the properties of the summatory function $S_{1}(N):=\sum_{n=0}^{N} l(n)$. The sequence $S_{1}(N)$ satisfies $S_{1}(0)=1$ and for every $m \geq 0$, 
$S_{\mathbf{1}}(4 m)=2 S_{\mathbf{1}}(m)-l(m)=S_{\mathbf{1}}(4 m+2), S_{\mathbf{1}}(4 m+1)=2 S_{\mathbf{1}}(m)-l(m)+l(2 m), S_{\mathbf{1}}(4 m+3)=2 S_{\mathbf{1}}(m)$.

Moreover, Lafrance, Rampersad and Yee showed that

$$
\limsup _{n \rightarrow+\infty} \frac{S_{\mathbf{1}}(n)}{\sqrt{n}}=\sqrt{2}, \liminf _{n \rightarrow+\infty} \frac{S_{\mathbf{1}}(n)}{\sqrt{n}}=\frac{\sqrt{3}}{3} .
$$

The sequences $S_{\mathbf{r}}(n)$ and $S_{\mathbf{1}}(n)$ are both 2-regular sequences (in the sense of Allouche and Shallit [1]). For the definition and properties of $k$-regular sequences, one can refer to [1]. Let $(s(n))_{n \geq 0}$ be a $k$-regular sequence over $\mathbb{Z}$. It was proved in [1] that there exists a constant $c$ such that $s(n)=O\left(n^{c}\right)$. In general, it is a difficult task to compute the exact growth order of sequences satisfying certain recursive relations such as $k$-regular sequences.

In [7], Gawron and Ulas obtained the sequence $\{a(n) \mid n \in \mathbb{N}\}:=\{m \in \mathbb{N} \mid c(m)=$ 1 ) where $(c(n))_{n \geq 0}$ is the sequence of coefficients of the compositional inverse of the generating function of the Thue-Morse sequence. The sequence $(a(n))_{n \geq 0}$ satisfies that $a(0)=0, a(1)=1, a(2)=2, a(3)=7$ and for all $n \geq 1, a(4 n+i)=a(4 n-1)+i+1$ with $i \in[0,2], a(8 n+3)=a(8 n)+7$ and $a(8 n+7)=4 a(4 n+3)+3$. They proved that

$$
\liminf _{n \rightarrow+\infty} \frac{a(n)}{n^{2}}=\frac{1}{6}, \limsup _{n \rightarrow+\infty} \frac{a(n)}{n^{2}}=\frac{1}{2}
$$

and $\left\{\frac{a(n)}{n^{2}}: n \geq 1\right\}$ is dense in $\left[\frac{1}{6}, \frac{1}{2}\right]$. In [2], Chen, Wen, Wu and the first author studied the maximal digit sum sequence $M_{\mathbf{r}}(n):=\max \left\{\sum_{j=i}^{i+n-1} r(j) \mid i \geq 0\right\}$ and proved that the abelian complexity $\rho(n)$ of the Rudin-Shapiro sequence satisfies $\rho(n)=M_{\mathbf{r}}(n)+1$ for every $n \geq 1$. It is remarkable that the authors in [2] just gave the recursive formulas for the sequence $M_{\mathbf{r}}(n)$ and proved the 2-regularity of the sequence $\rho(n)$. It is natural to ask whether the function $\rho(n)$ has similar properties as the summatory function $S_{\mathbf{r}}(n)$. In fact, it is of great interest to study the properties of sequences which satisfy certain recursive formulas.

This note focuses on the growth order of the abelian complexity $(\rho(n))_{n \geq 1}$ of the Rudin-Shapiro sequence $\mathbf{r}$. Firstly, by studying the maximal and minimal values of the function $\rho(n)$ in the interval $I_{k}:=\left[4^{k}, 4^{k+1}-1\right]$ with $k \geq 0$, we got $\rho(n)=\Theta(\sqrt{n})$. Then, we investigated two functions $s(k):=\min \{n \mid \rho(n)=k\}$ and $\ell(k):=\max \{n \mid \rho(n)=k\}$, and obtained the optimal lower and upper bound of the sequence $\left(\frac{\rho(n)}{\sqrt{n}}\right)_{n \geq 1}$. Finally, we showed that $\rho(n)$ is a quasi-linear function for 4 . As a consequence, the set $\left\{\frac{\rho(n)}{\sqrt{n}}: n \geq 1\right\}$ is dense between its optimal lower bound and upper bound. In detail, we proved the following theorems.

Theorem 1. For every integer $n \geq 1$, we have

$$
\sqrt{3} \leq \frac{\rho(n)}{\sqrt{n}} \leq 3
$$

Theorem 2. The set $\{\rho(n) / \sqrt{n}: n \geq 1\}$ is dense in $[\sqrt{3}, 3]$.

The outline of this note is as follows. In Section 2, we compute the maximal and minimal values of the function $\rho(n)$ in the interval $I_{k}:=\left[4^{k}, 4^{k+1}-1\right]$ for every $k \geq 0$. In Section 3, we give the proofs of Theorem 1 and Theorem 2.

\section{Basic Properties of the Function $\rho(n)$}

In this section, we exhibit some basic properties of the abelian complexity function $\rho(n)$ of the Rudin-Shapiro sequence $\mathbf{r}$. 
Following from ([2] Theorem 1 and Lemma 3), the abelian complexity function $\rho(n)$ of the Rudin-Shapiro sequence is given by the following formulas: $\rho(1)=2, \rho(2)=3$, $\rho(3)=4$ and for every integer $n \geq 1$,

$$
\begin{aligned}
\rho(4 n) & =2 \rho(n)+1, & & \rho(4 n+1)=2 \rho(n), \\
\rho(4 n+2) & =\rho(n)+\rho(n+1), & & \rho(4 n+3)=2 \rho(n+1) .
\end{aligned}
$$

Set $\rho(0):=1$. For every integer $n \geq 0$, let $\Delta \rho(n):=\rho(n+1)-\rho(n)$. Then $\Delta \rho(0)=$ $\Delta \rho(1)=\Delta \rho(2)=\Delta \rho(3)=1$, and for every integer $n \geq 1$,

$$
\begin{array}{r}
\Delta \rho(4 n)=-1, \Delta \rho(4 n+3)=1, \\
\Delta \rho(4 n+1)=\Delta \rho(4 n+2)=\Delta \rho(n) .
\end{array}
$$

This implies that $\Delta \rho(n) \in\{-1,1\}$ for every integer $n \geq 0$. The first 16 terms of $\rho(n)$, starting with $n=1$, are listed in Table 1 .

Table 1. The first 16 terms of the sequence $\rho(n)$.

\begin{tabular}{llll}
\hline$n$ & $\rho(n)$ & $n$ & $\rho(n)$ \\
\hline 1 & 2 & 9 & 6 \\
2 & 3 & 10 & 7 \\
3 & 4 & 11 & 8 \\
4 & 5 & 12 & 9 \\
5 & 4 & 13 & 8 \\
6 & 5 & 14 & 9 \\
7 & 6 & 15 & 10 \\
8 & 7 & 16 & 11 \\
\hline
\end{tabular}

For simplicity of notation, for every integer $k \geq 0$, put $m_{k}:=\frac{4^{k+1}-1}{3}, M_{k}:=4^{k+1}-1$ and $I_{k}:=\left[4^{k}, 4^{k+1}-1\right]$. Then we have the following two lemmas which give the minimal and maximal values of the function $\rho(n)$ in the interval $I_{k}$ for every $k \geq 0$.

Lemma 1. For every integer $k \geq 0$, the minimum value of $\rho(n)$ in $I_{k}=\left[4^{k}, 4^{k+1}-1\right]$ is $2^{k+1}$. Moreover,

$$
\max \left\{n \in I_{k}: \rho(n)=2^{k+1}\right\}=m_{k}
$$

Proof. We will prove this by induction on the variable $k$. For $k=0$, it follows from Table 1 that this assertion is true. Assume the assertion holds for the interval $I_{k}$.

We first show that $2^{k+2}$ is the lower bound for $\rho(n)$ in $I_{k+1}$. If $n$ lies in $I_{k+1}=$ $\left[4^{k+1}, 4^{k+2}-1\right]$, then we can write $n=4 m+d$ for some $m \in I_{k}$ and some $d \in\{0,1,2,3\}$. There are two cases to be considered.

1. When $4^{k} \leq m \leq 4^{k+1}-2$, (1) yields that for every $d \in\{0,1,2,3\}$

$$
\rho(n)=\rho(4 m+d) \geq 2 \min \left\{\rho(m): m \in I_{k}\right\}=2^{k+2} .
$$

The last equality is true under the inductive assumption.

2. When $m=4^{k+1}-1$, it follows from (2) that $\Delta(m)=1$, which implies

$$
\rho(m+1)=\rho\left(4^{k+1}-1\right)+1=\rho(m)+1 .
$$

Hence, for every $d \in\{0,1,2,3\}$, using (1) again, we have

$$
\rho(n)=\rho(4 m+d) \geq 2 \rho(m)>2 \min \left\{\rho(m): m \in I_{k}\right\}=2^{k+2} .
$$


At the same time, using the fact $m_{k+1}=4 m_{k}+1$, it is easy to check that

$$
\rho\left(m_{k+1}\right)=\rho\left(4 m_{k}+1\right)=2 \rho\left(m_{k}\right)=2^{k+2} .
$$

Now it suffices to show that

$$
m_{k+1}=\max \left\{n \in I_{k+1}: \rho(n)=2^{k+2}\right\} .
$$

Following from the inductive assumption, for every $m \in I_{k}$ satisfying $m>m_{k}$, we have $\rho(m) \geq \rho\left(m_{k}\right)+1$. By (1), we can get

$$
\begin{aligned}
& \rho\left(m_{k+1}+1\right)=\rho\left(4 m_{k}+2\right)=\rho\left(m_{k}\right)+\rho\left(m_{k}+1\right) \geq 2^{k+2}+1, \\
& \rho\left(m_{k+1}+2\right)=\rho\left(4 m_{k}+3\right)=2 \rho\left(m_{k}+1\right) \geq 2^{k+2}+2 .
\end{aligned}
$$

Now we only need to consider the case $n=4 m+d \geq 4 m_{k}+4$ with $d \in\{0,1,2,3\}$. In fact, for every $m_{k}+1 \leq m \leq 4^{k+1}-2$ and $d \in\{0,1,2,3\}$, it follows from (1) that

$$
\rho(4 m+d) \geq 2 \min \left\{\rho(m): m_{k}+1 \leq m \leq 4^{k+1}-1\right\} \geq 2^{k+2}+2 .
$$

By (3), the case $n=4 m+d$ for $m=4^{k+1}-1$ holds, which completes the proof.

Lemma 2. Let $k$ be a non-negative integer. The maximum value of $\rho(n)$ in $I_{k}=\left[4^{k}, 4^{k+1}-1\right]$ is $3 \cdot 2^{k+1}-2$ and this value occurs only at the point $n=M_{k}=4^{k+1}-1$.

Proof. We will prove this by induction on the variable $k$. For $k=0$, this assertion holds following from Table 1 . Assume the assertion is true for the interval $I_{k}$. When $n$ lies in $I_{k+1}$, let $n=4 m+d$ for some $m \in I_{k}$ and $d \in\{0,1,2,3\}$. Similarly with the proof of Lemma 1 , we divide it into two cases.

1. When $4^{k} \leq m<4^{k+1}-1$. By (1) and the inductive assumption, we have

$$
\rho(n)=\rho(4 m+d) \leq 2 \max \left\{\rho(m): m \in I_{k}\right\}+1<3 \cdot 2^{k+2}-2 .
$$

2. When $m=M_{k}=4^{k+1}-1$. Following from (1) and (2), we have

$$
\begin{aligned}
\rho\left(4 M_{k}\right) & =2 \rho\left(M_{k}\right)+1=3 \cdot 2^{k+2}-3, \\
\rho\left(4 M_{k}+1\right) & =2 \rho\left(M_{k}\right)=3 \cdot 2^{k+2}-4, \\
\rho\left(4 M_{k}+2\right) & =\rho\left(M_{k}\right)+\rho\left(M_{k}+1\right)=3 \cdot 2^{k+2}-3, \\
\rho\left(4 M_{k}+3\right) & =2 \rho\left(M_{k}+1\right)=3 \cdot 2^{k+2}-2 .
\end{aligned}
$$

This implies that $\rho(n) \leq \rho\left(4 M_{k}+3\right)=\rho\left(4^{k+2}-1\right)=3 \cdot 2^{k+2}-2$.

Following from (4) and (5), we can obtain that $M_{k+1}=4 M_{k}+3$ is the unique point in $I_{k+1}$ which attains the maximal value of $\rho$ in the interval $I_{k+1}$. This completes the proof.

Remark 1. From Lemma 1, we have that for every $n \geq 1$,

$$
\rho(n) \geq 2
$$

Remark 2. If $n \in I_{k}=\left[4^{k}, 4^{k+1}-1\right]$, Lemma 1 gives us

$$
\frac{\rho(n)}{\sqrt{n}}>\frac{2^{k+1}}{\sqrt{4^{k+1}}}=1,
$$

while Lemma 2 implies that

$$
\frac{\rho(n)}{\sqrt{n}}<\frac{3 \cdot 2^{k+1}-2}{\sqrt{4^{k}}}<6 .
$$


Thus, for every integer $n \geq 1,1 \leq \frac{\rho(n)}{\sqrt{n}} \leq 6$, and so $\rho(n)$ is roughly a constant times $\sqrt{n}$. However, these bounds are not optimal. Note that $\rho(n) \geq 2$ for every $n \geq 1$. It is easy to verify that

$$
\lim _{k \rightarrow \infty} \frac{\rho\left(M_{k}\right)}{\sqrt{M_{k}}}=\lim _{k \rightarrow \infty} \frac{3 \cdot 2^{k+1}-2}{\sqrt{4^{k+1}-1}}=3
$$

and

$$
\lim _{k \rightarrow \infty} \frac{\rho\left(m_{k}\right)}{\sqrt{m_{k}}}=\lim _{k \rightarrow \infty} \frac{2^{k+1}}{\sqrt{\frac{4^{k+1}-1}{3}}}=\sqrt{3} .
$$

In other words, 3 and $\sqrt{3}$ are two accumulation points of the set $\left\{\frac{\rho(n)}{\sqrt{n}}: n \geq 1\right\}$. In the following section, we will prove that 3 and $\sqrt{3}$ are the optimal upper and lower bound for the sequence $\left(\frac{\rho(n)}{\sqrt{n}}\right)_{n \geq 1}$ respectively.

\section{Proofs of Theorems 1 and 2}

Following that $M_{k}$ and $m_{k}$ both go to infinity with $k$ tending to infinity (by Lemmas 1 and 2), we can see that there are only finite number of places $n$ such that $\rho(n)$ has a fixed value $k$. When $\rho(n)=k$, for a fixed $k$, the ratio $\rho(n) / \sqrt{n}$ will be the smallest when $n$ is largest while it will be largest if $n$ is smallest. This leads us to the following idea: for a fixed $k \in \mathbb{N}$ with $k \geq 1$, let us focus on the smallest and largest values of $n$ such that $\rho(n)=k$. For this purpose, we introduced two auxiliary functions $s(k)$ and $\ell(k)$.

Definition 1. Given an integer $k \geq 1$, let $s(k)$ and $\ell(k)$ be the smallest and largest values of $n$ such that $\rho(n)=k$ respectively, i.e.,

$$
\begin{aligned}
& s(k):=\min \{n: \rho(n)=k\}, \\
& \ell(k):=\max \{n: \rho(n)=k\} .
\end{aligned}
$$

Following from (1), Table 1 and $\rho(0)=1$, the initial 8 terms of the sequences $s(k)$ and $\ell(k)$ are given in Table 2 .

Table 2. The initial values for the sequences $s(k)$ and $\ell(k)$.

\begin{tabular}{|c|c|c|c|c|c|c|c|c|}
\hline$k$ & 1 & 2 & 3 & 4 & 5 & 6 & 7 & 8 \\
\hline $\boldsymbol{s}(\boldsymbol{k})$ & 0 & 1 & 2 & 3 & 4 & 7 & 8 & 11 \\
\hline$\ell(k)$ & 0 & 1 & 2 & 5 & 6 & 9 & 10 & 21 \\
\hline
\end{tabular}

For the sequences $s(k)$ and $\ell(k)$, we have the following results.

Lemma 3. The sequence $\ell(k)$ satisfies $\ell(1)=0$ and for every integer $k \geq 1$,

$$
\ell(2 k)=4 \ell(k)+1, \quad \ell(2 k+1)=4 \ell(k)+2 .
$$

Proof. The assertion holds for $k=1$ by Table 2 . Fix some integer $k \geq 2$, assume $\ell(k)=n$. Then $\rho(n)=k$. By the definition of $\ell(n)$ and (2), for every integer $m>n, \rho(m) \geq \rho(n)+1=$ $k+1$ and

$$
\rho(n+1)=\rho(n)+1=k+1 .
$$

Therefore, for every $m>n$ and $d \in\{0,1,2,3\}$,

$$
\rho(4 m+d) \geq 2 \min \{\rho(m): m>n\}=2 k+2 .
$$


At the same time, when $m=n$, it is obvious that

$$
\begin{aligned}
\rho(4 n) & =2 \rho(n)+1=2 k+1, \\
\rho(4 n+1) & =2 \rho(n)=2 k, \\
\rho(4 n+2) & =\rho(n)+\rho(n+1)=2 k+1, \\
\rho(4 n+3) & =2 \rho(n+1)=2 k+2 .
\end{aligned}
$$

This implies that $\ell(2 k)=4 n+1=4 \ell(k)+1$ and $\ell(2 k+1)=4 n+2=4 \ell(k)+2$.

Lemma 4. The sequence $s(k)$ satisfies $s(1)=0, s(2)=1, s(3)=2$ and for every $k \geq 2$,

$$
s(2 k)=4 s(k)-1, \quad s(2 k+1)=4 s(k) .
$$

Proof. The initial values $s(1), s(2)$ and $s(3)$ can be easily verified by Table 2 . For an integer $k \geq 2$, suppose $s(k)=n$ for some integer $n$. Then $\rho(n)=k$. By the definition of $s(k)$ and (2), we have that $\rho(m)<\rho(n)$ whenever $m<n$ and

$$
\rho(n-1)=\rho(n)-1=k-1 .
$$

Therefore, following from (1), for every $0 \leq m<n-1$ and $d \in\{0,1,2,3\}$, we have

$$
\rho(4 m+d) \leq 2 \max \{\rho(m): 0 \leq m \leq n-1\}+1=2 k-1 .
$$

Note that the value of $4 m+d$ ranges from 0 to $4 n-5$. At the same time, it follows from (1) that

$$
\begin{aligned}
& \rho(4 n-4)=2 \rho(n-1)+1=2 k-1, \\
& \rho(4 n-3)=2 \rho(n-1)=2 k-2, \\
& \rho(4 n-2)=\rho(n-1)+\rho(n)=2 k-1, \\
& \rho(4 n-1)=2 \rho(n)=2 k .
\end{aligned}
$$

This implies that $s(2 k)=4 n-1=4 s(k)-1$. The other formula $s(2 k+1)=4 s(k)$ follows from the fact that $\rho(4 n)=2 \rho(n)+1=2 k+1$ and $\rho(i) \leq 2 k$ for every $0 \leq i \leq 4 n-1$. This ends the proof.

The following two propositions show the upper bound for the sequence $\ell(k)$ and the lower bound for the sequence $s(k)$. For the sake of simplicity, for every integer $k \geq 2$, let

$$
k=\sum_{j=0}^{m} k_{j} 2^{j}:=\left[k_{m} k_{m-1} \cdots k_{0}\right]_{2}
$$

be the binary expansion of $k$ with $m \geq 1$ and $k_{m}=1$. For every $x \geq 0$, let $\lfloor x\rfloor$ be the greatest integer which is no more than $x$.

Proposition 1. For every integer $k \geq 2$, we have

$$
\ell(k) \leq \frac{k^{2}}{3} .
$$

Proof. For every $k \geq 2$, let the binary expansion of $k$ be $\left[k_{m} k_{m-1} \cdots k_{0}\right]_{2}$. Following from Lemma 3, we have

$$
\ell(k)=\ell\left(\left[k_{m} k_{m-1} \cdots k_{0}\right]_{2}\right)=4 \ell\left(\left[k_{m} k_{m-1} \cdots k_{1}\right]_{2}\right)+k_{0}+1
$$


Now we apply (6) by replacing $k$ with $\left[k_{m} k_{m-1} \cdots k_{1}\right]_{2}$, which yields

$$
\ell\left(\left[k_{m} k_{m-1} \cdots k_{1}\right]_{2}\right)=4 \ell\left(\left[k_{m} k_{m-1} \cdots k_{2}\right]_{2}\right)+k_{1}+1 \text {. }
$$

Repeating this progress $m$ times, by the fact that $k_{j}=\left\lfloor\frac{k}{2^{j}}\right\rfloor-2\left\lfloor\frac{k}{2^{j+1}}\right\rfloor$ for every $0 \leq j \leq$ $m-1$, we can obtain that

$$
\begin{aligned}
\ell(k) & =\ell\left(\left[k_{m} k_{m-1} \cdots k_{0}\right]_{2}\right) \\
& =4 \ell\left(\left[k_{m} k_{m-1} \cdots k_{1}\right]_{2}\right)+k_{0}+1 \\
& =4^{2} \ell\left(\left[k_{m} k_{m-1} \cdots k_{2}\right]_{2}\right)+4\left(k_{1}+1\right)+k_{0}+1 \\
& =\cdots \cdots \\
& =4^{m} \ell\left(k_{m}\right)+\sum_{j=0}^{m-1} 4^{j}\left(k_{j}+1\right) \\
& =4^{m} \ell(1)+\sum_{j=0}^{m-1} 4^{j}\left(\left\lfloor\frac{k}{2^{j}}\right\rfloor-2\left\lfloor\frac{k}{2^{j+1}}\right\rfloor+1\right) \\
& =\frac{4^{m}-1}{3}+\sum_{j=0}^{m-1} 4^{j}\left(\left\lfloor\frac{k}{2^{j}}\right\rfloor-2\left\lfloor\frac{k}{2^{j+1}}\right\rfloor\right) \\
& \leq \frac{4^{m}}{3}+k-2\left\lfloor\frac{k}{2^{\prime}}\right\rfloor+4\left\lfloor\frac{k}{2^{2}}\right\rfloor-8\left\lfloor\frac{k}{2^{2}}\right\rfloor+\cdots+4^{m-1}\left\lfloor\frac{k}{2^{m-1}}\right\rfloor-2 \cdot 4^{m-1}\left\lfloor\frac{k}{2^{m}}\right\rfloor \\
& =\frac{4^{m}}{3}-2 \cdot 4^{m-1}+k+2 \sum_{j=1}^{m-1} 4^{j-1}\left\lfloor\frac{k}{2^{j}}\right\rfloor \\
& \leq \frac{4^{m}}{3}-2 \cdot 4^{m-1}+k+2 \sum_{j=1}^{m-1} 4^{j-1} \frac{k}{2^{j}} \\
& =2^{m-1} k-\frac{2}{3} \cdot 4^{m-1} .
\end{aligned}
$$

It suffices to show that

$$
2^{m-1} k-\frac{2}{3} \cdot 4^{m-1} \leq \frac{k^{2}}{3}
$$

Note that $2^{m} \leq k<2^{m+1}$. This implies that

$$
\frac{k}{4}<2^{m-1} \leq \frac{k}{2}
$$

Consider the function $f_{k}(x)=k x-\frac{2}{3} x^{2}$. It is not hard to check that $f_{k}(x)$ is strictly increasing on the interval $\left(\frac{k}{4}, \frac{k}{2}\right]$ with fixed $k \geq 1$. Hence

$$
k x-\frac{2}{3} x^{2} \leq f_{k}\left(\frac{k}{2}\right)=\frac{1}{3} k^{2}
$$

which is the desired result.

Proposition 2. For every integer $k \geq 2$, we have

$$
s(k) \geq \frac{k^{2}}{9}
$$

Proof. The assertion holds when $k=2$ and $k=3$ since $s(2)=1>4 / 9$ and $s(3)=2>1$. For every integer $k \geq 4$, let the binary expansion of $k$ be $\left[k_{m} k_{m-1} \cdots k_{0}\right]_{2}$ with $m \geq 2$ and $k_{m}=1$. Following from Lemma 4 , we have

$$
s(k)=s\left(\left[k_{m} k_{m-1} \cdots k_{0}\right]_{2}\right)=4 s\left(\left[k_{m} k_{m-1} \cdots k_{1}\right]_{2}\right)+k_{0}-1 .
$$


Arguing analogously as in the proof of Proposition 1, we have

$$
\begin{aligned}
& s(k)=s\left(\left[k_{m} k_{m-1} \cdots k_{0}\right]_{2}\right) \\
& =4^{m-1} s\left(\left[k_{m} k_{m-1}\right]_{2}\right)+\sum_{j=0}^{m-2} 4^{j}\left(k_{j}-1\right) \\
& =4^{m-1} s\left(2+k_{m-1}\right)+\sum_{j=0}^{m-2} 4^{j}\left(\left\lfloor\frac{k}{2^{j}}\right\rfloor-2\left\lfloor\frac{k}{2^{j+1}}\right\rfloor-1\right) \\
& =4^{m-1} s\left(2+k_{m-1}\right)-\frac{4^{m-1}-1}{3}+\sum_{j=0}^{m-2} 4^{j}\left(\left\lfloor\frac{k}{2^{j}}\right\rfloor-2\left\lfloor\frac{k}{2^{j+1}}\right\rfloor\right) \\
& =4^{m-1} s\left(2+k_{m-1}\right)-\frac{4^{m-1}-1}{3}-2 \cdot 4^{m-2}\left\lfloor\frac{k}{2^{m-1}}\right\rfloor+k+2 \sum_{j=1}^{m-2} 4^{j-1}\left\lfloor\frac{k}{2^{j}}\right\rfloor \\
& \geq 4^{m-1} s\left(2+k_{m-1}\right)-\frac{4^{m-1}}{3}-2 \cdot 4^{m-2}\left\lfloor\frac{k}{2^{m-1}}\right\rfloor+k+2 \sum_{j=1}^{m-2} 4^{j-1}\left(\frac{k}{2^{j}}-1\right) \\
& \geq 4^{m-1} s\left(2+k_{m-1}\right)-2 \cdot 4^{m-2}\left(3+k_{m-1}\right)+2^{m-2} k \\
& = \begin{cases}2^{m-2} k-2 \cdot 4^{m-2} & \text { if } k_{m-1}=0, \\
2^{m-2} k & \text { if } k_{m-1}=1\end{cases}
\end{aligned}
$$

The last equality uses the fact that $s(2)=1$ and $s(3)=2$.

1. If $k_{m-1}=0$, then we have $2^{m} \leq k<3 \cdot 2^{m-1}$, and it follows that

$$
\frac{k}{6}<2^{m-2} \leq \frac{k}{4}
$$

Put $g_{k}(x):=x k-2 x^{2}$. It is obvious that $g_{k}(x)$ is strictly increasing on the interval $\left(\frac{k}{6}, \frac{k}{4}\right]$ with fixed $k$. Hence

$$
2^{m-2} k-2 \cdot 4^{m-2} \geq g_{k}\left(\frac{k}{6}\right)=\frac{k^{2}}{9} .
$$

2. If $k_{m-1}=1$, then we have $3 \cdot 2^{m-1} \leq k<2^{m+1}$, and it follows that

$$
\frac{k}{8}<2^{m-2} \leq \frac{k}{6}
$$

which implies

$$
2^{m-2} k \geq \frac{k^{2}}{8} \geq \frac{k^{2}}{9}
$$

This ends the proof.

Now, combining Proposition 1 and Proposition 2, we can prove Theorem 1.

Proof of Theorem 1. For every integer $n \geq 1$, assume $\rho(n)=k$ for some integer $k$. It follows from Remark 1 that $k \geq 2$. By Proposition 1 and the definition of $\ell(k)$, we have

$$
\frac{\rho(n)}{\sqrt{n}} \geq \frac{k}{\sqrt{\ell(k)}} \geq \sqrt{3}
$$

Following from Proposition 2 and the definition of $s(k)$, we have

$$
\frac{\rho(n)}{\sqrt{n}} \leq \frac{k}{\sqrt{s(k)}} \leq 3 .
$$


This completes the proof.

By Remark 2 and Theorem 1, we get the following corollary.

Corollary 1. The numbers $\sqrt{3}$ and 3 are the optimal lower and upper bounds for the set $\left\{\frac{\rho(n)}{\sqrt{n}}\right.$ : $n \geq 1$ \} respectively.

To prove Theorem 2, we need an auxiliary notation which was firstly introduced in [8]. Let $b \geq 2$ be an integer and $f: \mathbb{N} \rightarrow \mathbb{Z}$ be a discrete function (or an integer sequence). For every $x \geq 0$, let

$$
\delta_{f}(x):=\limsup _{n \rightarrow+\infty} \frac{|f(n)|}{n^{x}} .
$$

Write $\Delta f(n):=f(n+1)-f(n)$. Set

$$
\alpha=\alpha(f):=\inf \left\{x \geq 0 \mid \delta_{f}(x)=0\right\} \text { and } \beta=\beta(f):=\inf \left\{x \geq 0 \mid \delta_{\Delta f}(x)=0\right\} .
$$

Definition 2. (Quasi-linear function) Let $f: \mathbb{N} \rightarrow \mathbb{Z}$. If $\alpha(f)>\beta(f)$ and there exists a constant $C_{1}>0$ and an integer $b \geq 2$ such that for all positive integers $n$ and $0 \leq i \leq b-1$,

$$
\left|f(b n+i)-b^{\alpha} f(n)\right| \leq C_{1} n^{\beta},
$$

then we call $f$ a quasi-linear function for $b$.

For the quasi-linear functions, we have the following lemma. For more details about this, see [8].

Lemma 5 ([8]). Given an integer function $f(n)$, set $\alpha:=\alpha(f)$. Suppose $a_{1}$ and $a_{2}$ are two accumulation points of $\left\{f(n) / n^{\alpha}: n \geq 1\right\}$. If $f$ is a quasi-linear function, then $\left\{f(n) / n^{\alpha}: n \geq 1\right\}$ is dense in $\left[a_{1}, a_{2}\right]$.

Proof of Theorem 2. Following from the fact that $\Delta \rho(n) \in\{-1,1\}$ for every $n \geq 0$, we have $\beta(\rho)=0$. By Theorem $1, \alpha(\rho)=\frac{1}{2}$. Moreover, for every $0 \leq i \leq 3$ and $n \geq 1$, (1) yields

$$
|\rho(4 n+i)-2 \rho(n)| \leq 2|\rho(n+1)-\rho(n)|=2 .
$$

Therefore $\rho(n)$ is a quasi-linear function for $b=4$.

It follows from Remark 2 that $\sqrt{3}$ and 3 are two accumulation points of the set $\{\rho(n) / \sqrt{n}: n \geq 1\}$. Hence we obtained the desired result by Lemma 5 .

Author Contributions: Formal analysis, X.L.; Investigation, X.L.; Project administration, X.L.; writing-review and editing, P.H. All authors have read and agree to the published version of the manuscript.

Funding: This work was supported by the National Natural Science Foundation of China (Grant No. 11801203).

Institutional Review Board Statement: Not applicable.

Informed Consent Statement: Not applicable.

Data Availability Statement: The data used to support the findings of this study are available from the corresponding author upon request.

Conflicts of Interest: The authors declare no conflict of interest. 


\section{References}

1. Allouche, J.-P.; Shallit, J. Automatic Sequences: Theory, Applications, Generalizations; Cambridge University Press: Cambridge, UK, 2003.

2. Lü, X.-T.; Chen, J.; Wen, Z.-X.; Wu, W. On the abelian complexity of the Rudin-Shapiro sequences. J. Math. Anal. Appl. 2017, 451, 822-838. [CrossRef]

3. Brillhart, J.; Morton, P. Uber Summen von Rudin-Shapiroschen Koeffizienten. Ill. J. Math. 1978, 22, 126-148. [CrossRef]

4. Brillhart, J.; Morton, P. A case study in mathematical research: The Golay-Rudin-Shapiro sequence. Am. Math. Mon. 1996, 130, 854-869. [CrossRef]

5. Brillhart, J. ; Erdős, P.; Morton, P. On sums of Rudin-Shapiro coefficients II. Pac. J. Math. 1983, 107, 39-69. [CrossRef]

6. Lafrance, P.; Rampersad, N.; Yee, R. Some properties of a Rudin-Shapiro-like sequence. Adv. Appl. Math. 2015, 63, 19-40. [CrossRef]

7. Gawron, M.; Ulas, M. On the formal inverse of the Prouhet-Thue-Morse sequence. Discret. Math. 2016, 339, 1459-1470. [CrossRef]

8. Lü, X.-T.; Chen, J.; Wen, Z.-X.; Wu, W. Limit behavior of the quasi-linear discrete functions. Fractals 2020, 28, 2050041. [CrossRef] 